\title{
Prolonged-Release (PR) Oxycodone/Naloxone Improves Bowel Function Compared with Oxycodone PR and Provides Effective Analgesia in Chinese Patients with Non-malignant Pain: A Randomized, Double-Blind Trial
}

Xiaomei Leng · Fengxiao Zhang · Shanglong Yao · Xisheng Weng • Kaizhi Lu • Gouzhong Chen •

Ming Huang · Yuguang Huang · Xiaofeng Zeng · Michael Hopp · Guodong Lu

Received: December 17, 2019 / Published online: February 3, 2020

(C) The Author(s) 2020

\begin{abstract}
Introduction: Prolonged-release oxycodone/ naloxone (OXN PR), combining an opioid analgesic with selective blockade of enteric $\mu$ opioid receptors, provided effective analgesia and improved bowel function in patients with moderate-to-severe pain and opioid-induced constipation in clinical trials predominantly
\end{abstract}

Enhanced Digital Features To view enhanced digital features for this article go to https://doi.org/10.6084/ m9.figshare.11663295.

Electronic supplementary material The online version of this article (https://doi.org/10.1007/s12325020-01244-x) contains supplementary material, which is available to authorized users.

X. Leng $\cdot$ X. Zeng $(\bowtie)$

Rheumatology and Immunology Department, Peking Union Medical College Hospital, Peking,

China

e-mail: xiaofeng.zeng@cstar.org.cn;

zengxiaofeng@medmail.com.cn

F. Zhang

Rheumatology and Immunology Department,

Hebei General Hospital, Shijiazhuang, China

S. Yao

Anesthesiology Department, Wuhan Union

Hospital, Tongji Medical College of Huazhong

University of Science and Technology, Wuhan, China conducted in Western countries. This doubleblind randomized controlled trial investigated OXN PR $(N=116)$ versus prolonged-release oxycodone (OXY PR, $N=115$ ) for 8 weeks at doses up to $50 \mathrm{mg} /$ day in patients with moderate-to-severe, chronic, non-malignant musculoskeletal pain and opioid-induced constipation recruited in China.

Methods: A total of 234 patients at least 18 years of age with non-malignant musculoskeletal pain for more than 4 weeks that was moderate-to-severe in intensity and required round-the-clock opioid therapy were randomized $(1: 1)$ to OXN PR or OXY PR. The primary endpoint was bowel function using the Bowel

X. Weng

Osteology Department, Peking Union Medical College Hospital, Peking, China

K. Lu

Anesthesiology Department, The First Affiliated Hospital of Third Military Medical University

(TMMU), Chongqing, China

G. Chen

Anesthesiology Department, Fuzhou General Hospital of Nanjing Military Command, Fuzhou, China

M. Huang

Anesthesiology Department, The General Hospital of Shenyang Military Region, Shenyang, China 
Function Index (BFI). Secondary endpoints included safety, Brief Pain Inventory-Short Form (BPI-SF), use of analgesic and laxative rescue medication, and health-related quality of life (EQ-5D).

Results: While BFI scores were comparable at baseline, at week 8 improvements were greater with OXN PR vs OXY PR (least squares mean [LSM] difference $(95 \% \mathrm{CI})-9.1(-14.0,-4.2)$; $P<0.001$. From weeks 2 to 8 , mean BFI scores were in the range of normal bowel function $(\leq 28.8)$ with OXN PR but were in the range of constipation $(>28.8)$ at all timepoints with OXY PR. Analgesia with OXN PR was similar and non-inferior to OXY PR on the basis of modified BPI-SF average $24-\mathrm{h}$ pain scores at week 8: LSM difference $(95 \% \mathrm{CI})-0.3(-0.5$, $-0.1) ; P<0.001$. The most frequent treatmentrelated AEs were nausea (OXN PR 5\% vs OXY PR $6 \%$ ) and dizziness (4\% vs $4 \%$ ).

Conclusion: OXN PR provided clinically meaningful improvements in bowel function and effective analgesia in Chinese patients with moderate-to-severe musculoskeletal pain and pre-existing opioid-induced constipation.

Trial Registration: ClinicalTrials.gov, identifier NCT01918098.

Keywords: Bowel function index; China; Musculoskeletal pain; Opioid-induced constipation; Pain; Prolonged-release oxycodone/ naloxone

\section{Key Summary Points}

Why carry out this study?

Prolonged-release, orally administered oxycodone/naloxone (OXN PR) has been shown to provide effective analgesia and improve bowel function in patients with opioid-induced constipation and moderate-to-severe non-cancer and cancer-related pain

As most patients enrolled in clinical trials of OXN PR were recruited in Western countries, additional data are needed from patients in other regions to help inform local treatment practices

This randomized controlled trial investigated improvements in bowel function, analgesia, and safety with OXN $\mathrm{PR}$ versus prolonged-release oxycodone (OXY PR) in patients with non-cancerrelated pain for more than 4 weeks and opioid-induced constipation who were recruited in China

\section{What was learned from the study?}

OXN PR was associated with comparable analgesia and greater improvements in Bowel Function Index scores vs OXY PR, and was generally well tolerated with no unanticipated adverse events in this population

OXN PR provided effective analgesia and clinically meaningful improvements in bowel function in Chinese patients with moderate-to-severe musculoskeletal pain and pre-existing opioid-induced constipation
Y. Huang

Anesthesiology Department, Peking Union Medical College Hospital, Peking, China

\section{Hopp}

Mundipharma Research GmbH \& Co.KG, Limburg, Germany

G. Lu

Mundipharma (China) Pharmaceutical Co. Ltd, Beijing, China

\section{INTRODUCTION}

Chronic pain affects approximately $22-36 \%$ of adults in China, a prevalence similar to that observed in Western countries [1-6]. Chronic, 
moderate-to-severe pain impacts numerous aspects of patients' physical and emotional well-being as well as impacting day-to-day activities $[1,7,8]$. Furthermore, the substantial economic burden arising as a result of absenteeism, reduced work productivity, and healthcare costs associated with chronic pain underscores the need for effective management approaches $[9,10]$.

Opioid analgesics can provide effective pain relief for carefully selected patients with moderate-to-severe pain who have not responded to other measures [11-13]. However, moderate-tosevere pain is often undertreated, in part because of inadequate assessment of pain, regulatory constraints, fear of addiction, and concerns regarding the side effects of opioids [14, 15]. Treatment guidelines recommend that the lowest effective opioid dose is prescribed, patients are informed about the risks and benefits of opioid therapy, and that efficacy, compliance, potential abuse or addiction, and side effects of treatment are regularly assessed [12, 13, 16]. Opioid-induced bowel dysfunction (OIBD) is a common class effect of opioid analgesics. OIBD arises when exogenous opioids interact with enteric $\mu$-opioid receptors which are located throughout the gastrointestinal tract. This results in the disruption of a variety of motility and secretory processes $[17,18]$. Symptoms can affect the entire gastrointestinal tract and include nausea, reflux, bloating, and opioid-induced constipation which is ranked by patients as the most bothersome symptom of OIBD $[19,20]$. Indeed, opioid-induced constipation can be so severe that many patients reduce the dosage or stop taking opioids to make it easier to have a bowel movement, thereby potentially sacrificing analgesia [19]. Laxatives aid defecation via localized effects on the colon and, given the unique etiology of opioid-induced constipation, it is unsurprising they fail to adequately address this condition in many patients $[21,22]$. Furthermore, laxatives can be associated with bothersome symptoms including flatulence and loss of bowel control [21].

Oxycodone is reported to be a more potent analgesic than morphine in some studies and, compared with immediate-release formulations, prolonged-release opioids can provide less fluctuation in plasma drug concentrations and support less frequent (twice-daily) dosing $[23,24]$. Prolonged-release, orally administered oxycodone/naloxone (OXN PR) was designed to address the underlying causes of OIBD by combining oxycodone with local selective blockade of enteric $\mu$-opioid receptors (naloxone). Orally administered naloxone can prevent or reverse opioid-induced constipation and owing to negligible systemic exposure (bioavailability $\leq 2 \%$ ) it does not impact the central, pain-relieving action of oxycodone $[25,26]$. Across a range of clinical trials and real-world observational studies, OXN PR has been demonstrated to provide effective analgesia and improve bowel function in patients with opioid-induced constipation and moderate-to-severe non-cancerand cancer-related pain [27]. Given that most patients enrolled in clinical trials of OXN PR were recruited in Western countries, additional data are needed from patients in other regions to help inform local treatment practices.

This randomized controlled trial investigated improvements in bowel function, analgesia, and safety with OXN PR versus prolonged-release oxycodone (OXY PR) in patients with noncancer-related pain for more than 4 weeks and opioid-induced constipation who were recruited in China.

\section{METHODS}

\section{Study Design}

Patients were enrolled from 32 hospitals in China into this phase III, double-blind study during routine clinical consultations (registered with ClinicalTrials.gov, NCT01918098). Patients at least 18 years of age were recruited with non-malignant musculoskeletal pain for more than 4 weeks that was moderate-to-severe in intensity ("average pain over last $24 \mathrm{~h}$ " $\geq 4$ points on $0-10$ scale) and were considered likely to benefit from round-the-clock opioid therapy ( $\geq 10-50 \mathrm{mg} /$ day OXY equivalent). Exclusion criteria included clinically significant cardiovascular, renal, hepatic, gastrointestinal or psychiatric disease, impaired liver or kidney function (aspartate aminotransferase, alanine 
aminotransferase, or alkaline phosphatase $>3 \times$ upper limit of normal [ULN], gamma-glutamyltranspeptidase $\geq 5 \times$ ULN, and total bilirubin or creatinine outside of reference range), recent or planned surgery that may affect gastrointestinal motility, and active alcohol or drug abuse (including individuals currently receiving opioid substitution therapy).

Following screening, eligible patients entered a run-in period (7-28 days) during which openlabel OXY PR administered orally, twice daily (BID), every $12 \mathrm{~h}$ (OxyContin ${ }^{\circledR}$, Bard Pharmaceuticals) was titrated to an effective analgesic dose and pre-study laxative was changed to bisacodyl (Dulcolax ${ }^{\circledR}$, Boehringer Ingelheim). Patients with constipation that was induced or worsened by opioid therapy and achieved stable pain control with OXY PR $10-50 \mathrm{mg} /$ day were randomized (1:1) to receive 8 weeks of oral, double-blind treatment with OXN PR (Targinact $^{\circledR}$, Bard Pharmaceuticals) or OXY PR BID at the OXY PR BID dose established during the runin phase. Treatment allocation was masked in a double-dummy fashion. Randomization was performed via the Oracle IRT system using a central randomization list generated by Mundipharma Research GmbH which was kept confidential (securely filed). Codes were not sitespecific. Patients were assigned to the next available code and randomized to treatment accordingly. Stratification was not used. The patient, investigator, and all personnel involved in the conduct and interpretation of the study were blinded to the medication codes. Study treatment was provided by the study sponsor and dispensed by delegated personnel to each patient during study visits. A 1-week safety follow-up was conducted at the end of doubleblind treatment or early study discontinuation.

During the study immediate-release morphine (10 mg, every 4-6 h; Qinghai Pharmaceuticals) was permitted as rescue medication for breakthrough pain and the laxative, bisacodyl (5-10 $\mathrm{mg}$ /day) was permitted at least $72 \mathrm{~h}$ after the last bowel movement (other laxatives were not allowed). If necessary, titration of study medication (OXN PR or OXY PR) was permitted up to $50 \mathrm{mg} /$ day. Patients who received OXY PR or OXN PR $50 \mathrm{mg} /$ day and consistently required more than two rescue medication doses of morphine were discontinued from double-blind treatment, as were those with no bowel movement following the rescue regimen of two doses of bisacodyl and an enema.

Study investigators were clinicians employed at the hospitals where the patients were recruited. Training for the study conduct was provided via site initiation meetings held at each study site and investigator meetings. Written standard operating procedures were implemented and monitored to ensure adherence to the study protocol, data accuracy, and compliance with Good Clinical Practices and Good Laboratory Practices. The study protocol was approved by the ethics committees at each institution (a list of the institutions with participating independent ethics committees is provided in the supplementary material), and the study was conducted in accordance with the Declaration of Helsinki, International Conference on Harmonization Guidelines for Good Clinical Practice, and China Good Clinical Practice guidelines. All patients provided written informed consent.

\section{Study Endpoints and Assessments}

The primary objective was to evaluate bowel function using the validated Bowel function Index $\left(\mathrm{BFI}^{1}\right)[28,29]$. BFI was assessed before randomization and at each study visit during double-blind treatment (days 1, 8, 15, 29, and 57) and was defined as the mean of three items: ease of defecation $(0=$ "easy/no difficulty" to $100=$ "severe difficulty"), feeling of incomplete bowel evacuation $(0=$ "not at all" to $100=$ "very strong"), and personal judgement of constipation $(0=$ "not at all" to $100=$ "very strong").

Pain was assessed as a secondary endpoint using the modified Brief Pain Inventory-Short Form (BPI-SF) [30]. Pain severity items included average pain over the last $24 \mathrm{~h}$, worst and least pain in the last $24 \mathrm{~h}$, and pain right now; pain interference (general activity, mood, walking

\footnotetext{
$\overline{1 \text { Copyright }}$ for the Bowel Function Index is owned by Mundipharma Laboratories $\mathrm{GmbH}$, Switzerland, 2002; the BFI is also the subject of European Patent Application Publication No. EP 1,860,988 and corresponding patents and applications in other countries.
} 
ability, work, relationships, sleep, and enjoyment of life) and relief provided by treatments in the last $24 \mathrm{~h}$ were also assessed. Patients also rated their average pain intensity over the last $24 \mathrm{~h}(0=$ "no pain" to $10=$ "pain as bad as you can imagine") each day in a diary. Other secondary endpoints included use of analgesic and laxative rescue medication (recorded daily in patient diaries) and health-related quality of life (EQ-5D scores were assessed at screening and end of double-blind treatment). Safety was monitored via vital signs, physical examination, hematology, blood chemistry, electrocardiograms, and adverse events (AEs), classified by Medical Dictionary for Regulatory Activities (MedDRA) system organ class and preferred terms. Opioid withdrawal was assessed on the basis of the modified Subjective Opiate Withdrawal Scale (SOWS; opioid withdrawal was defined as a score $>26$ ) [31]. Adherence to study medication was assessed by the study investigator (at each study visit patients were asked to return all used study medication containers and unused study medication).

\section{Statistical Analysis}

BFI was analyzed using mixed-model repeated measures (MMRM; treatment, site, time, and interaction between treatment and time were categorical variables and BFI pre-randomization value was a continuous variable) with least squares mean (LSM) difference in treatment groups at the end of the 8-week, double-blind treatment (day 57). The primary endpoint was assessed in the full analysis population (FAP: patients who received at least one dose of study medication and had at least one assessment during double-blind treatment). Supportive analyses were conducted in the per protocol population (PPP: patients who received at least one dose of study medication and complied with the study protocol). Sensitivity analyses using analysis of covariance (ANCOVA), adjusted for baseline value and site also compared BFI at day 57 between treatment groups in the FAP. BFI score at each study visit was summarized descriptively, with changes from baseline between study groups examined using exploratory two-sided $t$ tests. No formal imputation of missing data was done for the MMRM analysis, as it makes use of all available data, including study subjects with only partially complete data, to estimate the mean treatment effect. In the sensitivity analysis (using ANCOVA) comparing the BFI at day 57 (visit 7), the model used the last observation carried forward (LOCF) approach to impute missing data.

Post hoc analyses of BFI included descriptive statistics of non-constipated $(\mathrm{BFI} \leq 28.8)$ and constipated $(\mathrm{BFI}>28.8)$ patient subgroups (performed in the FAP and PPP) [29], descriptive statistics of the three individual BFI items at each visit (performed in the FAP and PPP), and sensitivity analysis using the MMRM model of the primary endpoint but excluding the BFI prerandomization value as a covariant factor (performed in the FAP).

Modified BPI-SF average pain over the last $24 \mathrm{~h}$ was analyzed at day 57 in the PPP using an MMRM model that included terms for treatment, site, time, pre-randomization value of modified BPI-SF average pain over the last $24 \mathrm{~h}$, and interaction between treatment and time. Sensitivity analysis for modified BPI-SF average pain over the last $24 \mathrm{~h}$ at day 57 used the same ANCOVA model as the primary endpoint, and tests for non-inferiority of OXN PR to OXY PR used a conservative margin of 0.8 for the LSM difference between treatments based on literature data for clinically meaningful differences on an 11-point numerical pain rating scale [32]. Safety was assessed in all patients who received at least one dose of double-blind study medication (safety population) and along with other secondary endpoints was summarized using descriptive statistics. Statistical testing utilized $t$ tests for continuous variables, chi-squared tests for categorical variables, was two-sided, and performed at the 5\% level of significance.

Sample size calculation was based on a superiority test of BFI. A 12-point difference in BFI between treatment groups was considered clinically meaningful, and an SD of 26 for BFI was presumed on the basis of prior studies $[28,31,33,34]$. A total of 200 evaluable patients in the FAP were needed to achieve $90 \%$ power at a significance level of 0.05 . Anticipating a $15 \%$ dropout rate, we planned to randomize 230 patients. 


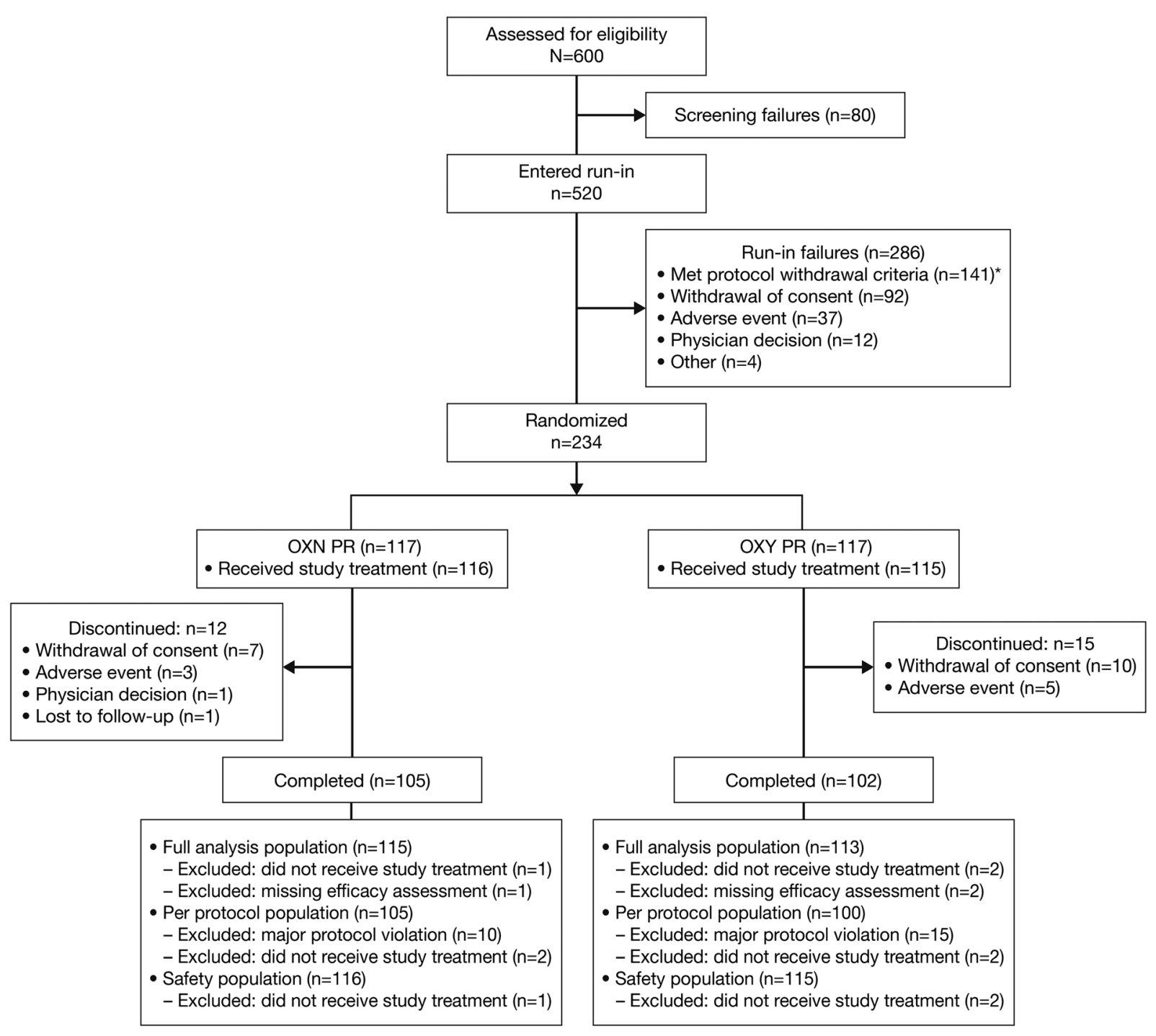

Fig. 1 Patient disposition. OXN PR prolonged-release oxycodone/naloxone, OXY PR prolonged-release oxycodone. Full analysis population comprised patients who received at least one dose of study medication and had at least one assessment during double-blind treatment; per protocol population comprised patients who received at

\section{RESULTS}

\section{Patient Disposition and Baseline Characteristics}

This study took place between September 2013 and November 2016, with 520 patients enrolled in the run-in phase and 234 randomized (1:1) to OXN PR or OXY PR (Fig. 1). Of the 141 patients

least one dose of study medication and complied with the study protocol; safety population comprised patients who received at least one dose of double-blind study medication. ${ }^{*} n=138$ due to not meeting constipation criteria and $n=3$ due to inadequate analgesia with OXY PR $50 \mathrm{mg} /$ day

who met protocol withdrawal criteria during the run-in phase, this was primarily due to not meeting constipation criteria $(n=138)$; only three patients did not receive adequate analgesia with OXY PR $50 \mathrm{mg} /$ day (Fig. 1). Overall, 207 patients (88\%) completed double-blind treatment. Within the low overall discontinuation rate the most frequent reason for discontinuation in both treatment groups was withdrawal 
Table 1 Baseline demographic and disease characteristics (full analysis population)

\begin{tabular}{|c|c|c|}
\hline Parameter & $\begin{array}{l}\text { OXN PR } \\
N=115\end{array}$ & $\begin{array}{l}\text { OXY PR } \\
(N=113)\end{array}$ \\
\hline Age, years; mean (SD) & $61.2(13.0)$ & $59.3(12.9)$ \\
\hline$<65$ years, $n(\%)$ & $69(60)$ & $75(66)$ \\
\hline $65-74$ years, $n(\%)$ & $28(24)$ & $22(20)$ \\
\hline$>74$ years, $n(\%)$ & $18(16)$ & $16(14)$ \\
\hline Female/male; $n(\%)$ & $\begin{array}{c}66(57) / 49 \\
(43)\end{array}$ & $\begin{array}{l}67(59) / 46 \\
(41)\end{array}$ \\
\hline BMI, $\mathrm{kg} / \mathrm{m}^{2}$; mean $(\mathrm{SD})$ & $24.1(3.5)$ & $23.7(3.3)$ \\
\hline \multicolumn{3}{|c|}{ Underlying pain condition; $n(\%)$} \\
\hline Arthralgia & $12(10)$ & $10(9)$ \\
\hline Back pain & $8(7)$ & $11(10)$ \\
\hline $\begin{array}{l}\text { Intervertebral disc } \\
\text { protrusion }\end{array}$ & $20(17)$ & $18(16)$ \\
\hline Musculoskeletal pain & $25(22)$ & $17(15)$ \\
\hline Osteoarthritis & $25(22)$ & $27(24)$ \\
\hline Osteoporosis & $10(9)$ & $6(5)$ \\
\hline Pain in extremity & $6(5)$ & $10(9)$ \\
\hline Periarthritis & $6(5)$ & $3(3)$ \\
\hline Other & $3(3)$ & $11(10)$ \\
\hline \multicolumn{3}{|c|}{ Prior analgesic medications, $n(\%)^{\mathrm{a}}$} \\
\hline $\begin{array}{l}\text { Anti-inflammatory and } \\
\text { anti-rheumatic products }\end{array}$ & $59(51)$ & $57(50)$ \\
\hline Opioids & $46(40)$ & $50(44)$ \\
\hline $\begin{array}{l}\text { Other analgesics/ } \\
\text { antipyretics }\end{array}$ & $18(16)$ & $12(11)$ \\
\hline
\end{tabular}

$B M I$ body mass index, $O X N P R$ prolonged-release oxycodone/naloxone, $O X Y P R$ prolonged-release oxycodone

${ }^{a}$ Patients may have received $>1$ prior analgesic medication

of consent, being slightly more common with OXY PR $[n=10(9 \%)]$ than OXN PR $(n=7$ $(6 \%)]$. No patient discontinued double-blind treatment as a result of meeting protocol withdrawal criteria. Baseline demographic and disease characteristics were balanced between treatment groups (Table 1). All patients were
Asian with a mean (SD) age of 60.3 (13.0) years (37\% were $\geq 65$ years). Slightly more female $(58 \%)$ than male $(42 \%)$ patients were enrolled. Osteoarthritis (23\%), musculoskeletal pain $(18 \%)$, and intervertebral disc protrusion (17\%) were the most common underlying pain conditions. Overall, $42 \%$ of patients had received opioid analgesia prior to study enrollment.

\section{Efficacy Outcomes: BFI Scores}

At baseline (day 1 of double-blind treatment) mean (SD) BFI scores were comparable in the OXN PR [55.5 (16.3)] and OXY PR [55.1 (16.2)] groups in the FAP. However, after 8 weeks of double-blind treatment the improvement in BFI score was significantly greater with OXN PR [23.4 (21.6)] versus OXY PR [34.8 (26.7)]; adjusted LSM difference (95\% CI) - $9.1(-14.0$, $-4.2) ; P<0.001$. This significantly superior effect of OXN PR versus OXY PR on BFI was also observed in the FAP after excluding seven patients (OXN PR $n=3$, OXN PR $n=4$ ) who were not constipated at baseline and thereby violated randomization criteria (adjusted LSM difference $\left[\begin{array}{ll}95 \% & \mathrm{CI}\end{array}\right]-9.5 \quad\left[\begin{array}{lll}-14.4, & -4.5\end{array}\right]$; $P<0.001$ ) and in the PPP (adjusted LSM difference $[95 \%$ CI $]-8.7 \quad\left[\begin{array}{lll}-13.9, & -3.5\end{array}\right]$; $P<0.001)$. Sensitivity analysis using the ANCOVA model supported the primary efficacy analysis: adjusted LSM difference $(95 \% \mathrm{CI})$ in BFI score between OXN PR and OXY PR at week 8 was $-10.5(-16.9,-4.0) ; P=0.002$. Post hoc sensitivity analyses of BFI at week 8 , performed using the same model as the primary analysis but without baseline BFI value as a covariant factor, also revealed a significantly greater improvement in BFI scores with OXN PR versus OXY PR (adjusted LSM difference [95\% CI $]-9.9[-15.1,-4.6] ; P<0.001)$.

Analysis of BFI scores at each study visit revealed decreases from baseline in both treatment groups with significantly greater improvement seen in patients receiving OXN $P R$ versus $O X Y P R$ from the first on-treatment study visit (day 8 ) to end of study treatment on day 57 ( $P \leq 0.01$; Fig. 2 ). From week 2 until the end of double-blind treatment, mean BFI scores were below the threshold for normal bowel 


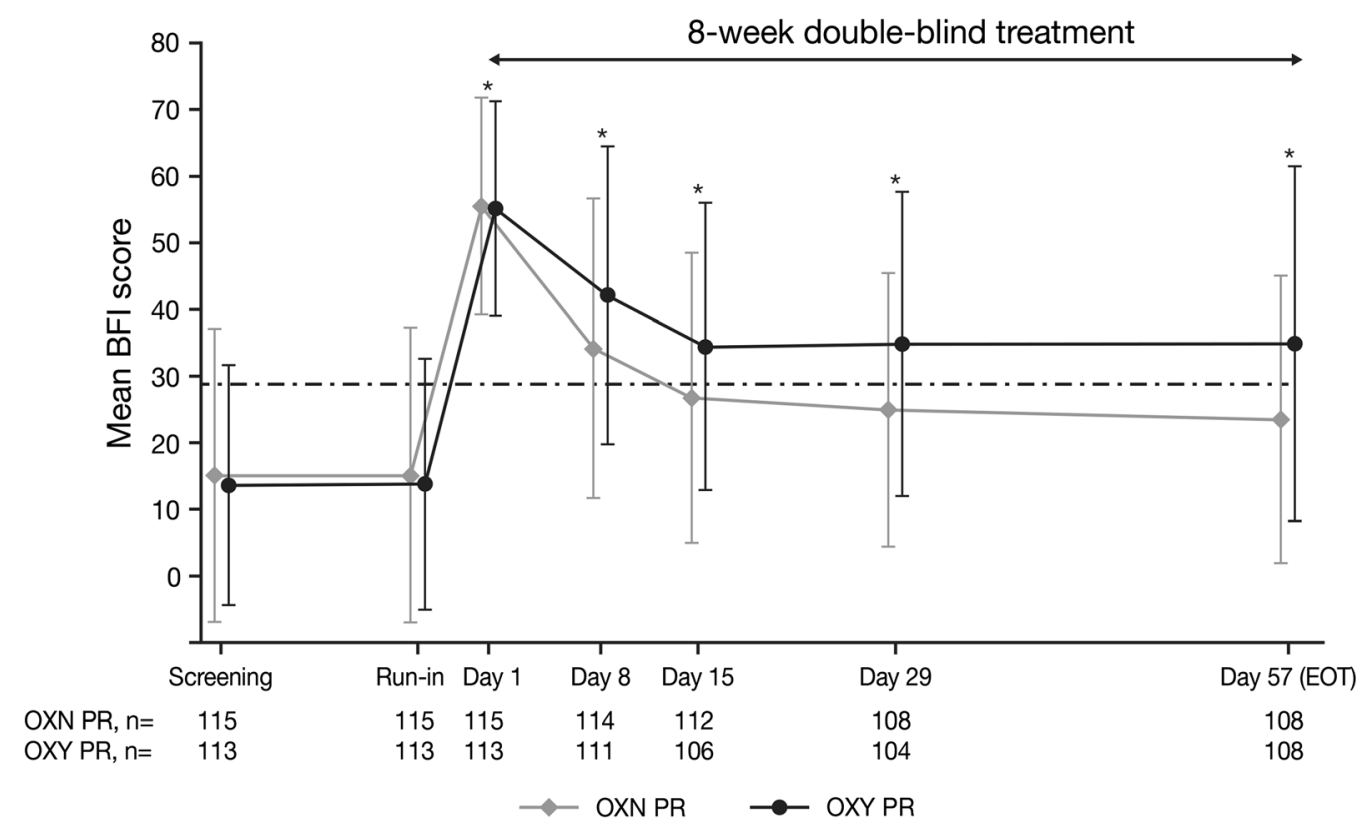

Fig. 2 Bowel Function Index Scores at each study visit (full analysis population). BFI Bowel Function Index, EOT end of study treatment, OXN PR prolonged-release oxycodone/naloxone, OXY PR prolonged-release oxycodone. Data are mean \pm SD. Lower scores indicate better bowel

function $(\leq 28.8)$ in patients randomized to OXN PR but mean BFI scores exceeded this threshold at all timepoints in those randomized to OXY PR (Fig. 2). A higher proportion of patients randomized to OXN PR versus OXY PR also had normalized bowel function (BFI $\leq 28.8$ ) within 1 week of study treatment $[42 \%$ $(n=48)$ vs $28 \%(n=31), P=0.026]$. This was maintained at each study visit during doubleblind treatment [week 2: $57 \%(n=64)$ vs $43 \%$ $(n=46), P=0.042$; week $4: 58 \%(n=63)$ vs $44 \%$ $(n=46), P=0.040$; week 8: $64 \%(n=69)$ vs $46 \%$ $(n=50) ; P=0.009]$. Comparable findings were observed in the PPP.

Post hoc analysis of the three individual BFI item scores also indicated greater reductions of constipation from baseline to week 8 with OXN $P R$ versus $O X Y$ PR (higher scores indicated worse constipation symptoms): mean (SD) decrease in "ease of defecation", - 34.0 (26.0) versus - 23.3 (29.7); "feeling of incomplete bowel evacuation", - 27.6 (26.2) versus - 13.8 (29.6); and "personal judgement of constipation", - 35.0 (25.7) versus - 22.9 (29.4). function. ${ }^{*} P \leq 0.01$ (two-sided $t$ test). EOT data could be included from patients without data at all study timepoints. Hashed line represents the BFI threshold of 28.8 for normal bowel function in patients with pain (higher scores indicated worse constipation symptoms) [29]

\section{Other Efficacy Outcomes}

In both treatment groups, modified BPI-SF average 24 -h pain scores decreased from prerandomization values and remained stable during double-blind treatment (Fig. 3). Analgesia provided by OXN PR was comparable and noninferior to OXY PR on the basis of modified BPISF average 24-h pain scores at week 8: LSM difference $(95 \% \quad \mathrm{CI}) \quad-0.3 \quad(-0.5,-0.1)$; $P<0.001$. Sensitivity analysis using ANCOVA also supported non-inferiority of OXN PR vs OXY PR for analgesia at week 8: LSM difference (95\% CI) $-0.3(-0.7,0.0) ; P<0.001$. The other three pain severity items of the modified BPI-SF also demonstrated comparable outcomes with OXN PR versus OXY PR ("pain right now", "worst pain in the last $24 \mathrm{~h}$ ", and "least pain in the last $24 \mathrm{~h}$ ") at week 8 (two-sided $t$ tests for non-inferiority, $P<0.001)$. There were also no notable differences between OXN PR and OXY $P R$ in the remaining pain interference and pain relief items of modified BPI-SF, except for "pain interference in mood" and "pain interference in 


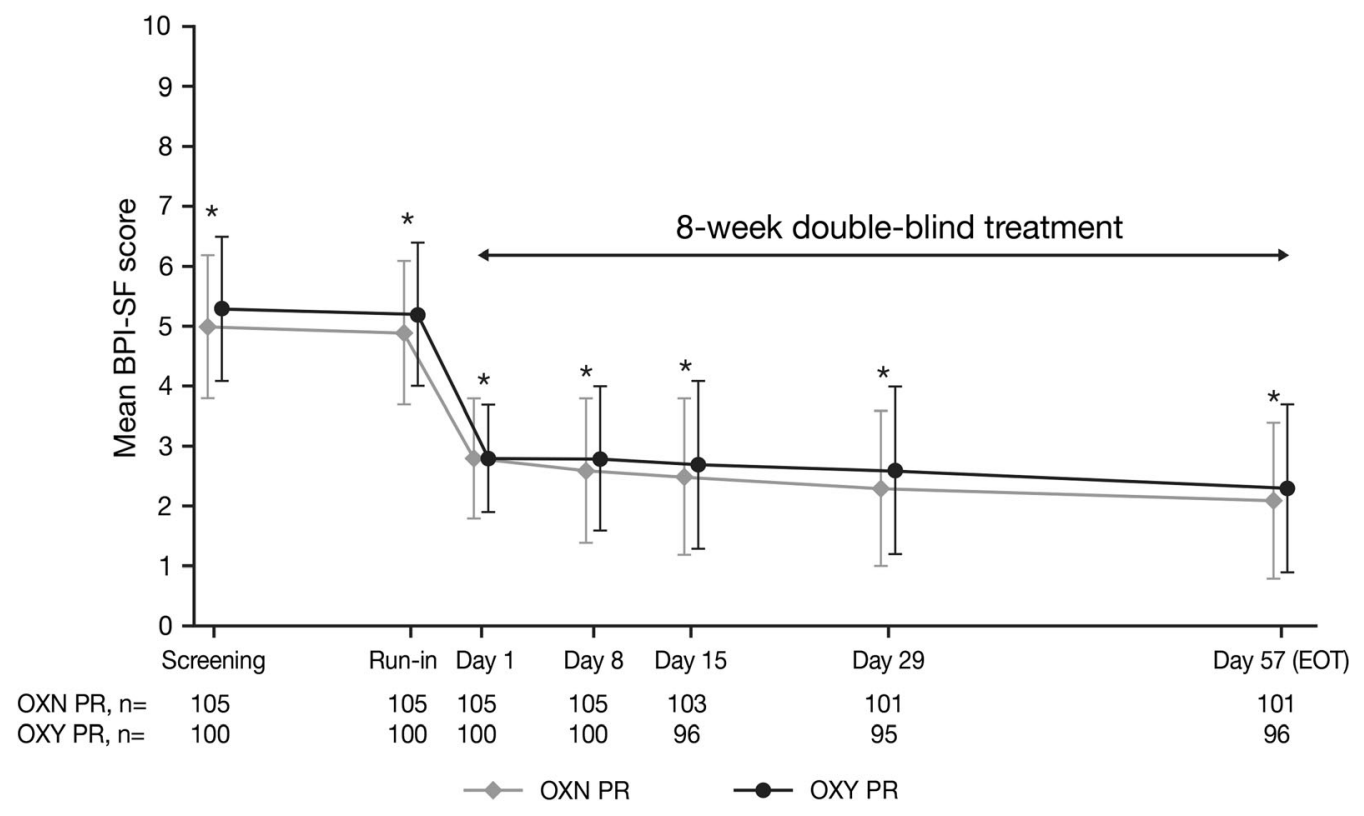

Fig. 3 Modified BPI-SF: average pain over the last $24 \mathrm{~h}$ (per protocol population). BPI-SF Brief Pain InventoryShort Form, EOT end of study treatment, OXN PR prolonged-release oxycodone/naloxone, OXY PR prolonged-release oxycodone. Data are mean \pm SD. Lower

sleep" at week 8 which slightly favored OXN PR vs OXY PR (exploratory $t$ tests; $P \leq 0.05$; Supplementary Table 1 ). Weekly average pain intensity, values based on daily reports in patient diaries, were similar for OXN PR and OXY PR throughout double-blind treatment (two-sided $t$ test for non-inferiority, $P<0.001$ ).

A similar proportion of patients randomized to OXN PR and OXY PR used analgesic rescue medication $[n=25$ of $115(22 \%)$ and $n=24$ of $113(21 \%)]$ and there were no notable differences in the proportion of patients who took rescue analgesia at each study visit. Median (range) total exposure to rescue analgesia during double-blind treatment was $0(0,1190) \mathrm{mg}$ and $0(0,1090) \mathrm{mg}$ in the OXN PR and OXY PR FAP, respectively. There was a trend for lower use of rescue laxative during double-blind treatment with OXN PR versus OXY PR (proportion of patients who took bisacodyl was $n=58$ of 115 , $50 \%$ versus $n=71$ of $113,63 \%$; $P>0.05$; median (range) exposure to bisacodyl was $5(0,270)$ $\mathrm{mg}$ vs $15(0,390) \mathrm{mg})$. There were no notable differences in mean (SD) EQ-5D scores scores indicate less pain. ${ }^{*} P \leq 0.01$ (two-sided $t$ test) for non-inferiority with a margin of 0.8 . BPI-SF scale: $0=$ no pain to $10=$ pain as bad as you can imagine. EOT data could be included from patients without data at all study timepoints

between patients randomized to OXN PR and OXY PR at baseline [62.7 (16.4) and 59.8 (17.7)] or at the end of double-blind treatment [76.3 (15.5) and 74.0 (15.5)], with most patients reporting no problems with mobility, self-care, usual activities, pain/discomfort, and anxiety/ depression, likely reflecting the good pain control achieved in both treatment groups.

\section{Safety Outcomes}

Mean (SD) exposure to study medication during double-blind treatment was consistent with the planned 8 weeks of treatment: OXN PR 59 (17) days and OXY PR 59 (19) days. Mean (SD) daily dose of study medication was comparable for OXN PR [21.3 (12.8) $\mathrm{mg}$ ] and OXY PR [20.3 (11.5) $\mathrm{mg}$ ] and reflected the mean (SD) daily doses of OXY PR established at the end of the run-in period [20 (12) $\mathrm{mg}$ and 18.9 (11) $\mathrm{mg}$, respectively].

Treatment-emergent AEs were reported by $45 \%$ (52 of 116) and 50\% (58 of 115) of patients randomized to $\mathrm{OXN} \mathrm{PR}$ and $\mathrm{OXY} P R$, 
respectively, and treatment-related AEs were reported by $16 \%$ (18 of 116) and $22 \%$ (25 of 115 ) of patients, respectively. Most AEs were of mild severity. The incidence of severe, allcausality $\mathrm{AE}$ was low and comparable between treatment groups: OXN PR 4\% (5 of 116) and OXY PR 6\% (7 of 115). Gastrointestinal disorders were reported less frequently with OXN PR (14\%; 16 of 116$)$ compared with OXY PR $(23 \%$; 26 of 115) while AEs from the system organ class "infections and infestations" were more common with OXN PR [11\% (13 of 116$)$ and 8\% (9 of 115), respectively]. As anticipated, the most frequently reported treatment-emergent AEs in both groups were nausea [OXN PR 6\% (7 of 116), OXY PR 8\% (9 of 115)], vomiting [OXN PR 6\% (7 of 116), OXY PR 8\% (9 of 115)], and dizziness [OXN PR 6\% (7 of 116), OXY PR 7\% (8 of 115)]. These were also the most frequent treatment-related AEs (Table 2). Six serious AEs (SAEs) were reported in five patients randomized to OXN PR and eight SAEs were reported by

Table 2 Treatment-related ${ }^{a}$ adverse events during doubleblind treatment ( $\geq 2 \%$, safety population)

\begin{tabular}{lll}
\hline $\begin{array}{l}\text { Adverse event, } \\
\boldsymbol{n}(\%)\end{array}$ & $\begin{array}{l}\text { OXN PR } \\
\boldsymbol{N}=\mathbf{1 1 6}\end{array}$ & $\begin{array}{l}\text { OXY PR } \\
(\boldsymbol{N}=\mathbf{1 1 5})\end{array}$ \\
\hline Nausea & $6(5)$ & $7(6)$ \\
Dizziness & $5(4)$ & $5(4)$ \\
Vomiting & $3(3)$ & $6(5)$ \\
$\begin{array}{l}\text { Abdominal } \\
\quad \text { discomfort }\end{array}$ & 0 & $3(3)$ \\
Pruritis & $2(2)$ & $1(1)$ \\
Somnolence & $2(2)$ & $1(1)$ \\
Abdominal pain, & 0 & $2(2)$ \\
$\quad$ upper & & $2(2)$ \\
Decreased appetite & 0 & $2(2)$ \\
Withdrawal & 0 & \\
$\quad$ syndrome & &
\end{tabular}

$O X N$ PR prolonged-release oxycodone/naloxone, $O X Y P R$ prolonged-release oxycodone

${ }^{\text {a }}$ Reasonable possibility of a causal relationship with study medication, per investigator assessment six patients randomized to OXY PR. No AE preferred term was reported by more than one patient and all SAEs were considered unrelated to study medication, except for withdrawal syndrome. This event occurred in a 58-year-old woman 1 day after the last dose of OXY PR and resolved following treatment with diazepam and tramadol. One death was reported during the study which occurred in an 87-year-old woman with an ongoing medical history of cerebral infarction, cardiomyopathy, hypertension, hypokalemia, and arterial occlusive disease and gangrene in her left foot. Her death was due to multiorgan failure and was not considered related to study medication (OXN PR). Twelve AEs in eight patients resulted in study discontinuation. Half these events were considered related to study medication, which included nausea, vomiting, dizziness, and depression with OXN PR, and muscle twitching and abdominal discomfort with OXY PR. Fewer patients randomized to OXN PR experienced AEs requiring dose interruption (6 AEs in 4 patients) compared with OXY PR (13 AEs in 6 patients).

Baseline modified SOWS were low and comparable for OXN PR [mean 3.6 (SD 3.1)] and OXY [3.8 (3.3)] and remained stable at week 1 [3.3 (3.7) and 3.3 (3.0)], indicating that switching in the run-in phase from prior analgesia to study medication did not result in drug withdrawal.

\section{DISCUSSION}

OXN PR was designed to address the underlying cause of opioid-induced constipation. While OXN PR has been widely investigated in Western populations and the clinical benefits of this treatment have also been demonstrated in patients from Korea with chronic pain, there are few data regarding the efficacy and safety of this analgesic in other Asian populations $[27,35,36]$. To our knowledge, this is the first study examining OXN PR in patients from China with moderate-to-severe non-cancer pain for more than 4 weeks and opioid-induced constipation. 
This randomized, double-blind, multicenter study met its primary endpoint, demonstrating that OXN PR significantly improved symptoms of opioid-induced constipation compared with OXY PR. While BFI scores were comparable between study groups at baseline, following 8 weeks of treatment adjusted LSM difference favored OXN PR: - 9.1 (95\% CI - 14.0, - 4.2), $P<0.001$. This finding was confirmed by sensitivity analyses. Significantly lower BFI scores with OXN PR versus OXY PR were observed from the first week of study treatment and maintained at each assessment timepoint until week 8 (end of double-blind treatment; $P$ $\leq 0.01$ ). Importantly, OXN PR resulted in normalization of bowel function for some patients: mean BFI scores were maintained below the threshold of 28.8 points from week 2 to week 8 , indicating normal bowel function [29]. In contrast, patients randomized to OXY PR had mean BFI scores of at least 34.4 throughout study treatment, indicative of ongoing constipation. The improved bowel function associated with OXN PR vs OXY PR was observed despite numerically lower use of laxatives.

The improved bowel function associated with OXN PR was not at the expense of effective pain control in this population of patients from China. Indeed, OXN PR provided similar analgesia to OXY PR, with average 24 -h pain scores below 3 (assessed on an 11-point scale) throughout double-blind treatment confirming stable and appropriate analgesic efficacy in this population. Other modified BPI-SF pain severity items were also non-inferior for OXN PR versus OXY PR, while "pain interference with mood" and "pain interference with sleep" favored OXN PR in an exploratory analysis, supporting the beneficial effects of combining oxycodone with naloxone. These findings of improved bowel function, quality of life, and effective pain control with OXN PR are consistent with numerous studies of patients with moderate-tosevere pain and opioid-induced constipation in Western countries and Korea [27, 35, 36]. OXN PR has also been shown to improve bowel function in Western patients with chronic, moderate-to-severe pain and ongoing opioidinduced constipation despite at least two classes of laxatives [37-40]. However, the present study was not designed to examine the impact of OXN PR in such patients from China with particularly difficult-to-treat opioid-induced constipation.

OXN PR was generally well tolerated in this study, with most patients receiving the planned 8 weeks of treatment (mean exposure to OXN PR was 59 days). No unanticipated side effects were detected and the most frequent allcausality AEs observed with both OXN PR and OXY PR of nausea ( $6 \%$ and $8 \%)$, vomiting ( $6 \%$ and $8 \%)$, and dizziness (6\% and 7\%) largely reflect the safety profile of these agents in randomized trials conducted in Western populations [33, 41, 42]. While the safety profiles of OXN PR and OXY PR in the present study were similar, the lower incidence of gastrointestinal events observed with the former (14\% versus $23 \%$ ) was anticipated as a result of selective blockade of enteric $\mu$-opioid receptors by naloxone.

This study was associated with several limitations including the restricted dose range of OXN PR (10-50 mg/day OXY equivalent) and relatively short treatment duration of 8 weeks, which reflect current opioid prescribing practices in China. OXN PR has been demonstrated to improve bowel function in Western patients with moderate-to-severe pain and opioid-induced constipation at daily doses up to $160 \mathrm{mg}$ without an analgesic ceiling effect and, in Europe, while OXN PR at a starting dose of $10 \mathrm{mg}$ twice daily is recommended, $160 \mathrm{mg} /$ day is approved for patients previously maintained on a stable daily dose who require greater analgesia $[43,44]$. Long-term follow-up studies have also shown that OXN PR can provide comparable analgesia and better bowel function versus OXY PR for up to 12 months of treatment [45]. As the present study was limited to patients with noncancer, musculoskeletal pain for more than 4 weeks, findings may not necessarily be extrapolated to patients with other types of pain. However, data from numerous randomized controlled trials conducted in other populations confirm that OXN PR can provide analgesia comparable to OXN PR while improving bowel function in selected patients with a wide variety of pain types including cancer-related pain, neuropathic pain, and 
Parkinson's disease-related pain, as well as for pain associated with osteoarthritis and other musculoskeletal disorders [27]. Finally, the design of the present trial did not permit identification of patients who may derive most benefit from OXN PR, or include a placebo arm, the latter reflecting the design of other recent studies evaluating OXN PR [27]. While the potential impact of a placebo effect on the analgesia observed could be queried, the twoarm active comparator design was considered appropriate because OXN PR was previously demonstrated to provide superior analgesia to placebo in a phase III, double-blind study of patients with moderate-to-severe chronic back pain [31]. Furthermore, addition of naloxone was shown not impact the analgesic efficacy of OXY PR in this study [31].

\section{CONCLUSION}

The results of this randomized, double-blind, multicenter study show OXN PR at doses up to $50 \mathrm{mg} /$ day provided clinically meaningful improvements in all measures of bowel function and effective analgesia in this population of Chinese patients with moderate-to-severe musculoskeletal pain and pre-existing opioidinduced constipation. In contrast to OXY PR, most patients who were randomized to OXN PR achieve normal bowel function by week 2 of treatment which was maintained throughout the study despite lower use of laxatives. OXN PR was generally well tolerated and there were no unanticipated side effects reported in this population. Compared with OXY PR, OXN PR was associated with fewer gastrointestinal AEs as well as beneficial effects on mood and sleep.

\section{ACKNOWLEDGEMENTS}

The authors thank the patients who participated in this study. The sponsor was overall responsible for the set-up, running, and reporting of the study. The study Investigators collected the data and the data was analyzed independently. The sponsor was not involved in collecting or analyzing the data.
Funding. This study was funded by Mundipharma (China) Pharmaceutical Co., Ltd. Fees for the journal's Rapid Service and Open Access Fees were funded by Mundipharma (China) Pharmaceutical Co., Ltd.

Medical Writing Assistance. Medical writing support was provided by Siân Marshall Ph.D. of SIANTIFIX Ltd, Cambridgeshire, UK, and was funded by Mundipharma Research Ltd.

Authorship. All named authors meet the International Committee of Medical Journal Editors (ICMJE) criteria for authorship for this article, take responsibility for the integrity of the work as a whole, and have given their approval for this version to be published.

Disclosures. Xiaomei Leng, Fengxiao Zhang, Shanglong Yao, Xisheng Weng, Kaizhi Lu, Gouzhong Chen, Ming Huang, Yuguang Huang, and Xiaofeng Zeng have nothing to disclose. Michael Hopp was an employee of Mundipharma Research GmbH \& Co.KG at the time this study was conducted (his current affiliation is Interdisciplinary Center Clinical Trials [IZKS], University Medical Center Mainz, Mainz, Germany). Guodong Lu is an employee of Mundipharma (China) Pharmaceuticals Co. Ltd.

Compliance with Ethics Guidelines. The study protocol was approved by the ethics committees at each institution (a list of the institutions with participating independent ethics committees is provided in the supplementary material), and the study was conducted in accordance with the Declaration of Helsinki, International Conference on Harmonization Guidelines for Good Clinical Practice, and China Good Clinical Practice guidelines. All patients provided written informed consent.

Data Availability. The datasets generated, analyzed, and reported within this manuscript may be requested in accordance with the Data Sharing Policy of Mundipharma Research Limited, available from www.mundipharma-rd.eu. 
Open Access. This article is licensed under a Creative Commons Attribution-NonCommercial 4.0 International License, which permits any non-commercial use, sharing, adaptation, distribution and reproduction in any medium or format, as long as you give appropriate credit to the original author(s) and the source, provide a link to the Creative Commons licence, and indicate if changes were made. The images or other third party material in this article are included in the article's Creative Commons licence, unless indicated otherwise in a credit line to the material. If material is not included in the article's Creative Commons licence and your intended use is not permitted by statutory regulation or exceeds the permitted use, you will need to obtain permission directly from the copyright holder. To view a copy of this licence, visit http://creativecommons.org/licenses/by$\mathrm{nc} / 4.0 /$.

\section{REFERENCES}

1. Breivik H, Collett B, Ventafridda V, Cohen R, Gallacher D. Survey of chronic pain in Europe: prevalence, impact on daily life, and treatment. Eur J Pain. 2006;10(4):287-333.

2. Chen B, Li L, Donovan C, et al. Prevalence and characteristics of chronic body pain in China: a national study. Springerplus. 2016;5(1):938.

3. Jackson T, Chen H, Iezzi T, Yee M, Chen F. Prevalence and correlates of chronic pain in a random population study of adults in Chongqing, China. Clin J Pain. 2014;30(4):346-52.

4. Johannes CB, Le TK, Zhou X, Johnston JA, Dworkin RH. The prevalence of chronic pain in United States adults: results of an Internet-based survey. J Pain. 2010;11(11):1230-9.

5. Reid KJ, Harker J, Bala MM, et al. Epidemiology of chronic non-cancer pain in Europe: narrative review of prevalence, pain treatments and pain impact. Curr Med Res Opin. 2011;27(2):449-62.

6. Wong WS, Fielding R. Prevalence and characteristics of chronic pain in the general population of Hong Kong. J Pain. 2011;12(2):236-45.

7. de Sola H, Salazar A, Duenas M, Ojeda B, Failde I. Nationwide cross-sectional study of the impact of chronic pain on an individual's employment: relationship with the family and the social support. BMJ Open. 2016;6(12):e012246.

8. Epstein RS, Teagarden JR, Cimen A, Sostek M, Salimi T. When people with opioid-induced constipation speak: a patient survey. Adv Ther. 2017;34(3): 725-31.

9. Gannon B, Finn DP, O'Gorman D, Ruane N, McGuire BE. The cost of chronic pain: an analysis of a regional pain management service in Ireland. Pain Med. 2013;14(10):1518-28.

10. Gaskin DJ, Richard P. The economic costs of pain in the United States. J Pain. 2012;13(8):715-24.

11. Chou R, Fanciullo GJ, Fine PG, et al. Clinical guidelines for the use of chronic opioid therapy in chronic noncancer pain. J Pain. 2009;10(2):113-30.

12. O'Brien T, Christrup LL, Drewes AM, et al. European Pain Federation position paper on appropriate opioid use in chronic pain management. Eur J Pain. 2017;21(1):3-19.

13. Dowell D, Haegerich TM, Chou R. CDC guideline for prescribing opioids for chronic pain: United States, 2016. JAMA. 2016;315(15):1624-45.

14. Cheung CW, Choo CY, Kim Y-C, et al. Collaborative efforts may improve chronic non-cancer pain management in Asia: findings from a ten-country regional survey. J Pain Relief. 2016;5:1000225.

15. Xia Z. Cancer pain management in China: current status and practice implications based on the ACHEON survey. J Pain Res. 2017;10:1943-52.

16. Manchikanti L, Kaye AM, Knezevic NN, et al. Responsible, safe, and effective prescription of opioids for chronic non-cancer pain: American Society of Interventional Pain Physicians (ASIPP) guidelines. Pain Physician. 2017;20(2S):S3-92.

17. Holzer P. Opioid receptors in the gastrointestinal tract. Regul Pept. 2009;155(1-3):11-7.

18. Sobczak M, Salaga M, Storr MA, Fichna J. Physiology, signaling, and pharmacology of opioid receptors and their ligands in the gastrointestinal tract: current concepts and future perspectives. J Gastroenterol. 2014;49(1):24-45.

19. Bell TJ, Panchal SJ, Miaskowski C, Bolge SC, Milanova T, Williamson R. The prevalence, severity, and impact of opioid-induced bowel dysfunction: results of a US and European patient survey (PROBE 1). Pain Med. 2009;10(1):35-42.

20. Cook SF, Lanza L, Zhou X, et al. Gastrointestinal side effects in chronic opioid users: results from a 
population-based survey. Aliment Pharmacol Ther. 2008;27(12):1224-32.

21. Emmanuel A, Johnson M, McSkimming P, Dickerson S. Laxatives do not improve symptoms of opioid-induced constipation: results of a patient survey. Pan Med. 2017;18:1932-40.

22. LoCasale RJ, Datto C, Margolis MK, Coyne KS. Satisfaction with therapy among patients with chronic non-cancer pain with opioid-induced constipation. J Manag Care Spec Pharm. 2016;22(3):246-53.

23. Thibault K, Calvino B, Rivals I, et al. Molecular mechanisms underlying the enhanced analgesic effect of oxycodone compared to morphine in chemotherapy-induced neuropathic pain. PLoS One. 2014;9(3):e91297.

24. Toyama K, Furuie H, Kuroda K, Ishizuka H. Pharmacokinetic bioequivalence studies of an extendedrelease oxycodone hydrochloride tablet in healthy Japanese subjects under fasting and fed conditions without an opioid antagonist. Drugs $R$ D. 2017;17(3):363-70.

25. Meissner W, Leyendecker P, Mueller-Lissner S, et al. A randomised controlled trial with prolonged-release oral oxycodone and naloxone to prevent and reverse opioid-induced constipation. Eur J Pain. 2009;13(1):56-64.

26. Smith K, Hopp M, Mundin G, et al. Naloxone as part of a prolonged release oxycodone/naloxone combination reduces oxycodone-induced slowing of gastrointestinal transit in healthy volunteers. Expert Opin Investig Drugs. 2011;20(4):427-39.

27. Morlion BJ, Mueller-Lissner SA, Vellucci R, et al. Oral prolonged-release oxycodone/naloxone for managing pain and opioid-induced constipation: a review of the evidence. Pain Pract. 2018;18(5): 647-65.

28. Rentz AM, Yu R, Muller-Lissner S, Leyendecker P. Validation of the Bowel Function Index to detect clinically meaningful changes in opioid-induced constipation. J Med Econ. 2009;12(4):371-83.

29. Ueberall MA, Muller-Lissner S, Buschmann-Kramm C, Bosse B. The Bowel Function Index for evaluating constipation in pain patients: definition of a reference range for a non-constipated population of pain patients. J Int Med Res. 2011;39(1):41-50.

30. Cleeland CS, Ryan KM. Pain assessment: global use of the Brief Pain Inventory. Ann Acad Med Singapore. 1994;23(2):129-38.

31. Vondrackova D, Leyendecker P, Meissner W, et al. Analgesic efficacy and safety of oxycodone in combination with naloxone as prolonged release tablets in patients with moderate to severe chronic pain. J Pain. 2008;9(12):1144-54.

32. Farrar JT, Young JP Jr, LaMoreaux L, Werth JL, Poole RM. Clinical importance of changes in chronic pain intensity measured on an 11-point numerical pain rating scale. Pain. 2001;94(2):149-58.

33. Ahmedzai SH, Nauck F, Bar-Sela G, Bosse B, Leyendecker P, Hopp M. A randomized, doubleblind, active-controlled, double-dummy, parallelgroup study to determine the safety and efficacy of oxycodone/naloxone prolonged-release tablets in patients with moderate/severe, chronic cancer pain. Palliat Med. 2012;26(1):50-60.

34. Löwenstein $\mathrm{O}$, Leyendecker $\mathrm{P}$, Hopp $\mathrm{M}$, et al. Combined prolonged-release oxycodone and naloxone improves bowel function in patients receiving opioids for moderate-to-severe non-malignant chronic pain: a randomised controlled trial. Expert Opin Pharmacother. 2009;10(4):531-43.

35. Hwang CJ, Chung SS, Lee KY, et al. Analgesic efficacy and safety of prolonged-release oxycodone/naloxone in Korean patients with chronic pain from spinal disorders. Clin Orthop Surg. 2018;10(1):33-40.

36. Lee KH, Kim TW, Kang JH, et al. Efficacy and safety of controlled-release oxycodone/naloxone versus controlled-release oxycodone in Korean patients with cancer-related pain: a randomized controlled trial. Chin J Cancer. 2017;36(1):74.

37. Jones GP, Tripathi SS. Oxycodone and naloxone combination: a 12-week follow-up in 20 patients shows effective analgesia without opioid-induced bowel dysfunction. Pain Ther. 2016;5(1):107-13.

38. Koopmans G, Simpson K, De AJ, Lux EA, Wagemans M, Van MY. Fixed ratio (2:1) prolonged-release oxycodone/naloxone combination improves bowel function in patients with moderate-to-severe pain and opioid-induced constipation refractory to at least two classes of laxatives. Curr Med Res Opin. 2014;30(11):2389-96.

39. Mehta V, Alaward S, Kuravinakop S, Nikolic S. Effect of a fixed-dose opioid agonist/antagonist on constipation in patients on long-term opioids for non-malignant pain unable to tolerate laxatives. Pain Physician. 2014;17(5):415-24.

40. Poelaert J, Koopmans-Klein G, Dioh A, et al. Treatment with prolonged-release oxycodone/naloxone improves pain relief and opioid-induced constipation compared with prolonged-release oxycodone in patients with chronic severe pain and laxativerefractory constipation. Clin Ther. 2015;37(4): 784-92. 
41. Löwenstein O, Leyendecker P, Lux EA, et al. Efficacy and safety of combined prolonged-release oxycodone and naloxone in the management of moderate/severe chronic non-malignant pain: results of a prospectively designed pooled analysis of two randomised, double-blind clinical trials. BMC Clin Pharmacol. 2010;10:12.

42. Simpson K, Leyendecker P, Hopp M, et al. Fixedratio combination oxycodone/naloxone compared with oxycodone alone for the relief of opioid-induced constipation in moderate-to-severe noncancer pain. Curr Med Res Opin. 2008;24(12): 3503-12.

43. Dupoiron D, Stachowiak A, Loewenstein O, et al. A phase III randomized controlled study on the efficacy and improved bowel function of prolonged-release (PR) oxycodone-naloxone (up to $160 / 80 \mathrm{mg}$ daily) vs oxycodone PR. Eur J Pain. 2017;21:1528-37.

44. Napp Pharmaceuticals. Targinact Summary of Product Characteristics 2017 [updated 13 January 2017. http://www.medicines.org.uk/emc/medicine/ 22908.

45. Blagden M, Hafer J, Duerr H, Hopp M, Bosse B. Long-term evaluation of combined prolonged-release oxycodone and naloxone in patients with moderate-to-severe chronic pain: pooled analysis of extension phases of two phase III trials. Neurogastroenterol Motil. 2014;26(12):1792-801. 